\title{
CLINICAL IMAGES \\ Severe bilateral vision loss due to a unilateral penetrating injury by sympathetic ophthalmitis
}

\author{
L M van Zyl
}

The patient sustained an accidental injury that resulted in broken glass causing a penetrating eye injury in her left eye. A corneal laceration with iris prolapse was not identified at a secondary hospital. Eight weeks after the injury, she presented at Groote Schuur Hospital complaining of decreased vision and severe pain in both eyes. The diagnosis was sympathetic ophthalmitis after all infective and inflammatory causes were excluded. Her visual acuities were counting fingers at $30 \mathrm{~cm}$ with her left eye and 6/60 in her right eye. On cursory examination, the left eye seemed normal (Fig. 1) but a corneal scar with prolapsing intra-ocular tissue was visible on elevating the upper lid (Fig. 2). She was treated with systemic immunosuppression (intravenous methylprednisolone) and topical corticosteroids. The prolapsed iris was excised and the corneal laceration repaired. Vision in the left eye did not improve but improved to $6 / 36$ in the right eye.

\section{Discussion}

Sympathetic ophthalmitis is a rare ocular inflammatory condition where a penetrating injury or intra-ocular surgery to one eye predisposes the other, uninjured, eye to vision loss. The incidence is $0.19 \%$ following penetrating eye injuries and $0.007 \%$ following intra-ocular surgery. ${ }^{1}$ It is classically a panuveitis but may present with anterior segment inflammation only. ${ }^{2}$ It presents within 3 months of injury to the exciting eye and is thought to be a cellmediated auto-immune response to uveal antigen ${ }^{1,2}$ isolated from the lymphatic system and exposed to the blood circulatory system during penetrating injuries and intra-ocular surgery. ${ }^{2}$ Sympathetic ophthalmitis is prevented by early repair of a penetrating eye injury or enucleation within 2 weeks of injury. Long-term immunosuppression is indicated as the condition is known to flare up even 60 years after an eye injury. ${ }^{2}$

Sympathetic ophthalmitis could have been avoided in this case, highlighting that ocular injuries should be assessed thoroughly and referred to an ophthalmologist when in doubt. Ocular injuries with sharp objects should carry a high index of suspicion that the globe might be penetrated. Penetrating eye injuries should be referred

Lourens van Zyl is a registrar in the Division of Ophthalmology at Groote Schuur Hospital, Cape Town.

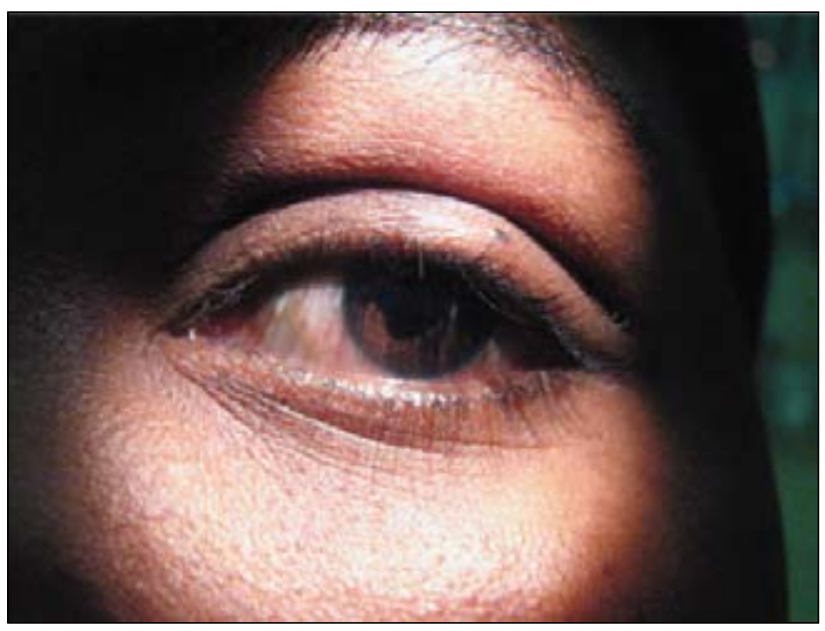

Fig. 1. The patient's eye looks normal in the primary position.

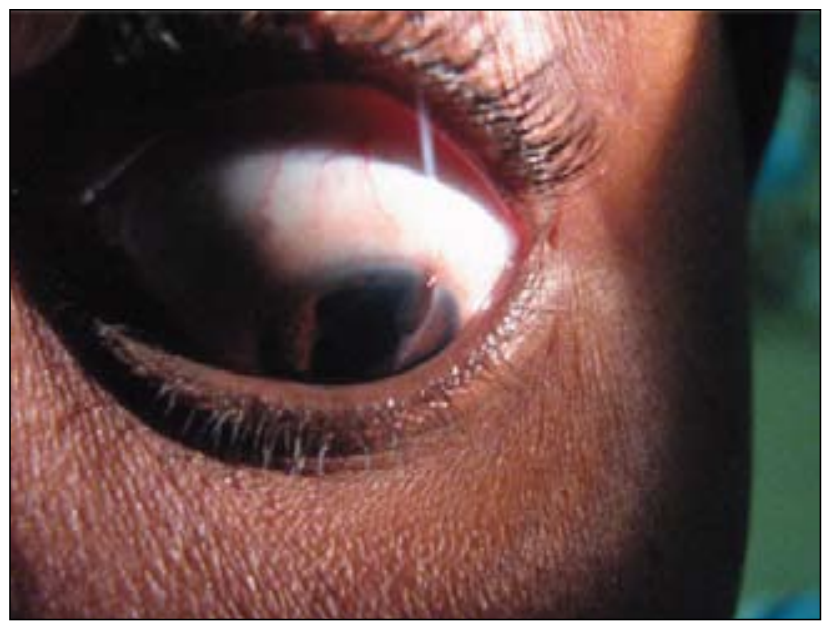

Fig. 2. Lifting the upper eyelid reveals a corneal laceration with uveal prolapse.

with an eye shield and not a pad, to prevent pressure on the globe that might cause extrusion of intra-ocular contents. No topical drops or ointment should be applied as the preservatives are toxic to the exposed intra-ocular tissue. Glass injuries of the cornea may be subtle; the irregular shape of the pupil may be the only visible sign that the globe is penetrated. 